\title{
CORRECTION
}

Open Access

\section{Correction to: New insight for metformin against bladder cancer}

Amr Ahmed EL-Arabey ${ }^{1,2}$

\section{Correction}

The author noted a few errors in the original article [1] need correction. The correct content is below.

1. The 3rd paragraph in background should be removed due to duplication.

2. In page 2 under Anti-carcinogenic effects of metformin, line 15 , the 'in vitro and in vitro' should be 'in vitro and in vivo'.

Received: 10 July 2018 Accepted: 13 July 2018

Published online: 24 July 2018

\section{Reference}

1. EL-Arabey AA. New insight for metformin against bladder cancer. Genes and Environment. 2017;39(13) https://doi.org/10.1186/s41021-017-0074-z. 